\title{
DOI: $10.53469 /$ jcmp.2022.04(02).04 \\ Efficacy of Gegenqinlian Xiezhuo Decoction in the Treatment of Non-alcoholic Fatty Liver of Spleen Deficiency and Blood Stasis Syndrome
}

\author{
Xiaoqian Wang ${ }^{1}$, Huaizhen Liu ${ }^{2, *}$ \\ ${ }^{1}$ Anhui University of Traditional Chinese Medicine \\ ${ }^{2}$ First Affiliated Hospital of Anhui University of Traditional Chinese Medicine \\ *Corresponding author, $1639152001 @ q q . c o m$
}

\begin{abstract}
Objective: To explore the clinical efficacy of Gegenqinlian Xiezhuo Decoction in the treatment of non-alcoholic fatty liver disease with spleen deficiency and blood stasis. Methods: A total of 62 patients with non-alcoholic fatty liver disease of spleen deficiency and blood stasis type admitted to the outpatient and ward of our hospital from October 2020 to June 2021 were selected and randomly divided into two groups with 31 cases in each group. The control group was treated with polyene phosphatidylcholine capsules orally, and the treatment group was treated with Gegen Qinlian Xiezhuo decoction on the basis of the control group. The therapeutic effects of the two groups were compared: (1) Liver function indexes: alanine aminotransferase (ALT), aspartate aminotransferase (AST) and glutamyl transferase (GGT). (2) Glucose and lipid metabolism indexes: fasting blood glucose (FBG), fasting insulin (FINS), insulin resistance index (HOMA-IR), triglyceride (TG), and total cholesterol (TC). Results: The total effective rate of the treatment group was $93.54 \%$, which was significantly higher than $67.74 \%$ of the control group, and the difference was statistically significant $(P<0.05)$. After treatment, the levels of AST, ALT and GGT in the treatment group were lower than those in the control group, and the difference was statistically significant (P<0.05). After treatment, the levels of FBG, FINS, HOMA-IR, TG and TC in the treatment group were lower than those in the control group, and the differences were statistically significant $(P<0.05)$. Conclusion: Gegenqinlian Xiezhuo Decoction has definite curative effect in treating NAFLD of spleen deficiency and blood stasis type, which can significantly improve the condition, improve liver function indexes, regulate blood glucose and blood lipid, and has good clinical safety.
\end{abstract}

Keywords: Gegenqinlian Xiezhuo Decoction, Non-alcoholic fatty liver disease, Spleen stasis turbidity type, Liver function, Blood glucose and blood lipid.

\section{Introduction}

Non-alcoholic fatty liver disease (NAFLD) is a chronic liver disease with a high prevalence worldwide. It is the largest chronic liver disease in China. If not treated in time, it may lead to cirrhosis, liver fibrosis and hepatocellular carcinoma, and endanger the life safety of patients[1]. The occurrence of this disease is relatively hidden, and the course of disease is slow. Most patients are asymptomatic at the early stage, or only have symptoms such as fatigue, epigastric fullness, and rib pain. Therefore, it is easy to be missed and misdiagnosed in clinic, and the best time for early intervention treatment is missed. The pathogenesis of NAFLD is complex. Insulin resistance[2], mitochondrial dysfunction[3], endoplasmic reticulum stress[4], fatty acids[5], intestinal flora[6] and dietary structure can affect the pathogenesis of NAFLD[7]. No specific drugs for NAFLD have been approved for clinical use[8]. However, in recent years, traditional Chinese medicine has been well developed in the treatment of this disease, with obvious curative effect and small side effects. In this study, patients with NAFLD of spleen deficiency and blood stasis turbidity were treated with Gegen Qinlian Xiezhuo Decoction, aiming to explore its clinical effect, in order to provide some reference for the treatment of NAFLD.

\section{Information and Methodology}

\subsection{General Information}

62 patients with non-alcoholic fatty liver disease of spleen deficiency and blood stasis type admitted to the outpatient and ward of our hospital from October 2020 to June 2021 were selected and randomly divided into two groups with 31 cases in each group. The control group was treated with polyene phosphatidylcholine capsules orally, and the treatment group was treated with Gegen Qinlian Xiezhuo decoction on the basis of the control group. There were 19 males and 12 females in the treatment group; 38 to 64 years old, with an average age of $(53.58 \pm 3.94)$ years old; 21 males and 10 females in the control group; they were 37 to 69 years old with an average age of $(53.60 \pm 3.92)$ years old. This study was approved by the Ethics Committee. There was no significant difference in general data between the two groups $(\mathrm{P}>0.05)$.

\subsection{Diagnostic Criteria}

1) Western diagnostic criteria

Reference "Guidelines for the prevention and treatment of nonalcoholic fatty liver disease (updated 2018)"[9] and "Guidelines for the diagnosis and treatment of nonalcoholic fatty liver disease"'[10]. (1) Clinical manifestations: occult pain in the liver area, fullness of the hypochondriac rib, general fatigue, and loss of appetite. (2) Signs: The common sign of NAFLD is hepatomegaly, and a few patients may have mild jaundice; liver cirrhosis signs include liver face, jaundice, splenomegaly, palm of liver, spider nevus, ascites and lower limb edema, abdominal vein exposure, male breast development, skin pigmentation, etc.

\section{2) TCM syndrome differentiation standard}

Reference "nonalcoholic fatty liver disease diagnosis and treatment of integrated traditional Chinese and western 
medicine consensus opinion (2017)"[11] spleen deficiency and blood stasis turbidity syndrome differentiation standard. Main symptoms: (1) Threatened rib pain, mainly stabbing or dull pain; (2) The lump under the hypochondrium; (3) Diet fatigue; (4) fat and full abdomen. Secondary symptoms: (1) epigastric fullness; (2) cough, expectoration and salivation; (3) nausea anorexia greasy; (4) Limbs are trapped. Tongue pulse: tongue fat with tooth marks or ecchymosis, greasy moss, fine or astringent pulse. With the above two main symptoms and sub-symptoms 1 or 2 , reference to the tongue pulse, can be clear syndrome differentiation.

\subsection{Inclusion and Exclusion Criteria}

\section{1) Inclusion criteria}

(1) In line with the above diagnostic criteria of Western medicine and TCM; (2) atients aged 30 to 70 years; (3) Patients could be treated with polyene phosphatidylcholine capsules and Gegenqinlian Xiezhuo Decoction; (4) Patients voluntarily received treatment and participated in the study.

\section{2) Exclusion criteria}

(1) Exclusion of alcoholic hepatitis and other liver diseases; (2) excluding patients with severe diseases such as heart, brain, lung, kidney and hematopoietic system; (3) excluding patients with hyperlipidemia; (4) excluding patients with hematopoietic and metabolic diseases; (5) excluding patients during pregnancy and lactation; (6) excluding patients with mental disorders and mental diseases.

\subsection{Criteria for Elimination, Shedding and Suspension of Cases}

\section{1) Exclusion and exclusion criteria}

(1) Serious violation of inclusion or exclusion criteria, should not be random; (2) Those who did not use drugs after randomization; (3) no post-treatment visit data; (4) Any other serious violation of the scheme that affects the efficacy judgment and safety judgment. The final elimination of cases is determined by the main researchers and data management personnel during verification.

\section{2) Suspension test standards}

(1) Serious safety problems occurred in the test should be suspended in time; (2) The test found that the drug treatment effect is poor, does not have clinical value, and should stop the test.

\subsection{Treatment}

Control group: oral polyene phosphatidylcholine capsules (Sanofi (Beijing) Pharmaceutical Co., Ltd.; h20059010), 3 times a day, 2 grains each time, $228 \mathrm{~g}$ each grain, and with diet and exercise guidance. Observation group: on the basis of + traditional Chinese medicine (Gegen Qinlian Xiezhuo Fang based on addition and subtraction).

(1) The composition of Gegen Qinlian Xiezhuo prescription: Radix Pseudostellariae 15g, Radix Puerariae 25g, Rhizoma
Atractylodis Macrocephalae 20g, Rhizoma Pinelliae 9g, Flos Carthami 10g, Radix Scutellariae 15g, Rhizoma Coptidis 9g, Bupleurum 10g, Radix Paeoniae Rubra 12g; the right side rib fullness add fragrant aconite, tulip each10g; abdominal distension, Magnolia officinalis, Fructus aurantii each 10g; fatigue shortness of breath plus astragalus $30 \mathrm{~g}$, Poria $15 \mathrm{~g}$; right flank walking pain plus toosendanzi, yanhusuo, perfume each $10 \mathrm{~g}$.

(2) Traditional Chinese medicine is made by the first affiliated hospital of Anhui University of Traditional Chinese Medicine decoction room, each dose of decoction about $300 \mathrm{ml}$, divided into two bags, morning and evening after $30 \mathrm{~min}$ warm.

Both groups were treated for 4 weeks, and 3 courses were observed for 12 weeks.

\subsection{Observation Indicators}

The clinical efficacy, liver function indexes, glycolipid metabolism indexes and adverse reactions were compared between the two groups. (1) Clinical efficacy: After the end of treatment, the main clinical symptoms basically disappeared, then it was judged to be cured; after the end of treatment, the main clinical symptoms of the disease were significantly improved, it was determined to be markedly effective; after the end of treatment, the main clinical symptoms of the disease have improved, it is judged to be effective; after the end of treatment, the main clinical symptoms of the disease did not improve significantly, or even aggravate, then judged invalid; total effective rate of treatment $=$ cure rate + significant efficiency + effective rate. (2) Liver function indexes: alanine aminotransferase (ALT), aspartate aminotransferase (AST) and glutamine transferase (GGT). (3) Glucose and lipid metabolism indexes: fasting blood glucose (FBG), fasting insulin (FINS), insulin resistance index (HOMA-IR), triglyceride (TG), and total cholesterol (TC). (4) Adverse reactions: including abdominal pain, diarrhea, rash, headache and other discomforts.

\subsection{Statistical Method}

The data in this study were analyzed and processed by SPSS26.0 statistical software. The measurement data such as liver function index and glucose and lipid metabolism index were expressed as $(\mathrm{x} \pm \mathrm{s})$. The $\mathrm{t}$ test and clinical efficacy were expressed as rate (\%). The $\chi 2$ test was used, $\mathrm{P}<0.05$, indicating that the difference was statistically significant.

\section{Results}

\subsection{Comparison of Liver Function Indexes Before and after Treatment Between the Two Groups}

Liver function indexes of the two groups were significantly decreased after treatment, and the observation group was lower than the control group $(\mathrm{P}<0.05)$, as shown in Table 1 .

\subsection{Comparison of Glucose and Lipid Metabolism Indexes Between the Two Groups Before and After Treatment}

After treatment, the blood lipid levels of the two groups were significantly decreased, and the observation group was lower 
than the control group $(\mathrm{P}<0.05)$, as shown in Table 2 .

3.3 Comparison of Comprehensive Efficacy between the Two Groups the total effective rate in the treatment group was $93.54 \%$, which was significantly higher than $67.74 \%$ in the control group, and the difference was statistically significant $(\mathrm{P}<0.01)$, as shown in Table 3.

Table 1: Comparison of liver function indexes between the two groups before and after treatment $(\bar{x} \pm s, U / L)$

\begin{tabular}{cccccc}
\hline Time & Group & $\mathrm{n}$ & ALT & AST & GGT \\
\hline \multirow{2}{*}{ Before treatment } & Treatment group & 31 & $59.01 \pm 15.71$ & $51.86 \pm 11.72$ & $58.95 \pm 10.56$ \\
& Control group & 31 & $57.29 \pm 14.03$ & $46.74 \pm 9.72$ & $56.18 \pm 13.11$ \\
After treatment & Treatment group & 31 & $35.36 \pm 6.73$ & $27.81 \pm 7.99$ & $31.94 \pm 9.47$ \\
& Control group & 31 & $43.43 \pm 9.46$ & $37.82 \pm 8.85$ & $47.21 \pm 11.99$ \\
\hline
\end{tabular}

Table 2: Comparison of glucose and lipid metabolism indexes between two groups before and after treatment $(\overline{\mathrm{x}} \pm \mathrm{s})$

\begin{tabular}{|c|c|c|c|c|c|c|c|}
\hline Time & Group & $\mathrm{n}$ & FBG $(\mathrm{mmol} / \mathrm{L})$ & FINS (pmol $/ \mathrm{mL})$ & HOMA-IR & $\mathrm{TG}(\mathrm{mmol} / \mathrm{L})$ & $\mathrm{TC}(\mathrm{mmol} / \mathrm{L})$ \\
\hline Before & Treatment group & 31 & $5.49 \pm 0.90$ & $17.51 \pm 4.29$ & $5.09 \pm 1.14$ & $3.01 \pm 0.27$ & $4.08 \pm 0.45$ \\
\hline treatment & Control group & 31 & $5.51 \pm 1.11$ & $\begin{array}{c}16.83 \pm \\
4.30\end{array}$ & $5.07 \pm 1.03$ & $3.00 \pm 0.26$ & $4.17 \pm 0.50$ \\
\hline After treatment & $\begin{array}{l}\text { Treatment group } \\
\text { Control group }\end{array}$ & $\begin{array}{l}31 \\
31\end{array}$ & $\begin{array}{l}4.36 \pm 0.84 \\
5.20 \pm 0.84\end{array}$ & $\begin{array}{l}12.33 \pm 3.17 \\
15.45 \pm 4.07\end{array}$ & $\begin{array}{l}3.25 \pm 0.94 \\
4.46 \pm 1.53\end{array}$ & $\begin{array}{l}1.21 \pm 0.11 \\
1.92 \pm 0.26\end{array}$ & $\begin{array}{l}3.37 \pm 0.42 \\
4.03 \pm 0.44\end{array}$ \\
\hline
\end{tabular}

Table 3: Comparison of comprehensive efficacy between the two groups $(\bar{x} \pm s)$

\begin{tabular}{ccccccc}
\hline Group & $\mathrm{n}$ & Cure & Effectual & Effective & Invalid & Total treatment rate \\
\hline Treatment group & 31 & 7 & 13 & 9 & 2 & $29(93.54)$ \\
Control group & 31 & 3 & 8 & 10 & 10 & $21(67.74)$ \\
Z & & & & & -2.684 \\
P & & & & & 0.007 \\
\hline
\end{tabular}

\subsection{Security Analysis}

There were no serious adverse events and drug-related adverse reactions in both groups during the experiment.

\section{Analysis}

The pathogenesis of NAFLD in western medicine has developed from "secondary strike theory" to "multiple strike theory". In recent years, key risk factors including overweight, insulin resistance, sedentary lifestyle and dietary pattern changes, genetic factors and intestinal barrier dysfunction have been identified. Intervention measures to reduce weight and change diet can help reduce the incidence of NAFLD. Non-alcoholic fatty liver disease (NAFLD) is first manifested as liver fat accumulation (steatosis), and then develops into non-alcoholic steatohepatitis (NASH), liver inflammation, fibrosis, cirrhosis and hepatocellular carcinoma[12] . NAFLD is common in up to $80 \%$ of obese patients and more than $60 \%$ of diabetic patients. Cardiovascular disease is the main cause of death, followed by extrahepatic cancer, and then liver-specific complications of cirrhosis and hepatocellular carcinoma. Studies have shown[13] that dietary fat and cholesterol independently promote steatosis, cholesterol crystal accumulation and inflammatory corpuscle activation through different but complementary pathways, inducing or aggravating the occurrence of this disease. Changes in lifestyle are still the main intervention for NAFLD. Weight loss through diet adjustment and exercise can lead to histological improvement and reversal of metabolic complications[14]. The commonly used drugs in clinic mainly include statins (lipid-lowering), metformin (insulin enhancer) and thiazolidinediones (hypoglycemic). However, these drugs have some adverse reactions. For example, long-term use of simvastatin can cause side effects such as liver damage and rhabdomyolysis, characterized by elevated transaminases and creatine kinase.

However, there is no unified name for this disease in traditional Chinese medicine. According to its clinical manifestations or etiology and pathogenesis, it is classified into many diseases and syndromes[15], such as "accumulation", "phlegm turbidity", "hypochondriac pain", "hepatopathy" and so on. Most of them believe that emotional disorder, improper diet, excessive fatigue, body deficiency, obesity and so on are the main causes of this disease. Traditional Chinese medicine treatment starts from the whole, combines disease differentiation and syndrome differentiation, and takes liver, spleen and kidney as the core. Starting from qi, phlegm, dampness, blood stasis and deficiency, clinical treatment is based on deficiency and excess. Because the spleen is the main transport, the main lifting, and is the source of qi and blood biochemistry and the hub of body fluid distribution, if the spleen is too strong, the valley will become active and nutritious; on the contrary, it leads to qi and blood can't be biochemical, transportation and transformation disorders, subtle can't be transported into blood and become water wet turbidity evil, condensed in the body into phlegm and fat and fatty liver. In addition, the liver in the pathological state of laxative function is significantly affected, can further damage the spleen and stomach function, and the more serious lipid turbidity, the faster the deterioration of the disease. Therefore, endogenous phlegm-dampness and spleen dysfunction are the pathogenesis of this disease, and the basic principles should be invigorating spleen and nourishing yin, removing blood stasis and resolving turbidity[16]. The nature of the disease is deficiency and excess, deficiency and excess mixed, excess for qi stagnation, blood stasis, phlegm, deficiency for viscera function loss, weak qi and blood[17]. Therefore, in the treatment should adhere to syndrome differentiation and treatment, seize the focus and core.

Gegenqinlian Xiezhuo Decoction is based on Gegenqinlian Decoction in Treatise on Febrile Diseases, and it is combined with the addition and subtraction of major therapeutic methods based on dispelling dampness and removing blood stasis. It has good clinical efficacy for non-alcoholic fatty liver disease, which can effectively reduce liver lipid 
deposition, restore blood lipid and liver function, and delay the development of the disease. In the prescription, kudzu root rises the qi of clearing yang in spleen and stomach, produces body fluid to stop thirst, and prince ginseng nourishes yin and produces body fluid, which is a monarch drug. Atractylodis Macrocephalae Jianpizaoshi, radix paeoniae rubra scattered blood stasis soft liver, Pinellia drying wet phlegm, descending inverse Xiaopi, so that wet spleen exuberance, phlegm no birth; huanglian Qingrezaoshi, the source of phlegm, a total of subjects; bamboo ru clearing heat phlegm trouble, and half summer, a warm one cool, strengthen the function of phlegm; chaihushan Tiaoda liver qi and soothe liver depression; scutellaria bitter cold clearing heat helps Huanglian clear gastrointestinal damp heat, safflower, red peony root promoting blood circulation and removing blood stasis Tongluo with adjuvant drugs, compatibility of various drugs, set spleen, phlegm, removing blood stasis as one, both symptoms and root treatment, cold and warm use, attack but not steep, not stagnation. Combined with clinical symptoms dialectical addition and subtraction, flexible. Modern pharmacological studies have shown that Radix Puerariae can improve insulin resistance, regulate glucose and lipid metabolism, improve oxidative stress, reduce inflammatory response, and regulate intestinal flora structure[18]. Scutellaria baicalensis can regulate the important factors of liver lipid synthesis and metabolism, down-regulate fat-producing genes, thereby reducing liver lipid deposition, and activate relevant signaling pathways to improve insulin resistance, and treat NAFLD[19]. Radix Paeoniae Rubra has the effects of regulating blood glucose and lipid, anti-inflammatory and antibacterial, anti-tumor, anti-oxidation, and regulating immunity[20]. Traditional Chinese medicine monomer has been proved to be able to treat NAFLD by regulating inflammation, lipid production, insulin sensitivity, mitochondrial dysfunction, autophagy and intestinal microflora[21].

The data of this study showed that the total effective rate of the treatment group was $93.54 \%$, which was significantly higher than that of the control group $(67.74 \%)$, and the difference was statistically significant $(\mathrm{P}<0.05)$. The levels of ALT, AST, GGT, FBG, FINS, HOMA-IR, TG and TC in the treatment group were lower than those in the control group, and the difference was statistically significant $(\mathrm{P}<0.05)$, indicating that Gegenqinlian Xiezhuo Decoction had remarkable therapeutic effect on non-alcoholic fatty liver disease. In summary, the clinical application of Gegenqinlian Xiezhuo Decoction in the treatment of NAFLD due to spleen deficiency and blood stasis is helpful to improve liver function and regulate glucose and lipid metabolism. It has high clinical efficacy and small side effects, which is worthy of clinical application.

\section{References}

[1] Cai Piao, Song Li, Liu Jiaxin, Tang Song Qi, Huang Wei. Effect of compatibility of meridian-introduction drugs on the mechanism of Xuefu Zhuyu Decoction in preventing non-alcoholic fatty liver disease[J]. Journal of Beijing University of Traditional Chinese Medicine, 2021, 44 (03): 252-258.

[2] Sun Hongshuang, Li Penglin, Liu Yongshuang, He Chuncheng, Gao Lingna. Effects of curcumin on liver
$11 \beta$-HSD1 expression and insulin resistance in rats with non-alcoholic fatty liver disease[J]. Chinese Journal of Experimental Zoology, 2021, 29 (05): 664-669.

[3] Xu Zijin, Lin Susu, Tong Zheren, Chen Suhong, Cao Yifeng, Li Qiaoqiao, Jiang Yuli, Cai Weijie, Tong Yingpeng, Zahra Bathaie S, Wang Ping. Crocetin ameliorates non-alcoholic fatty liver disease by modulating mitochondrial dysfunction in L02 cells and zebrafish model[J]. Journal of Ethnopharmacology, 2021, 285.

[4] Wang Mengxiao, Li Bingbing, Qin Fujian, Ye Junmei, Jin Liang. Obesity induced Ext1 reduction mediates the occurrence of NAFLD $[\mathrm{J}]$. Biochemical and Biophysical Research Communications, 2022, 589:

[5] Ngamlerst Chattraya, Udomkasemsab Arunwan, Kongkachuichai Ratchanee, Kwanbunjan Karunee, Chupeerach Chaowanee, Prangthip Pattaneeya. The potential of antioxidant-rich Maoberry (Antidesma bunius) extract on fat metabolism in liver tissues of rats fed a high-fat $\operatorname{diet}[\mathrm{J}]$. BMC Complementary and Alternative Medicine, 2019, 19(1).

[6] Li Mengci, Rajani Cynthia, Zheng Xiaojiao, Jia Wei. The microbial metabolome in metabolic-associated fatty liver disease $[\mathrm{J}]$. Journal of Gastroenterology and Hepatology, 2021:

[7] Gerges Samar H., Wahdan Sara A., Elsherbiny Doaa A., El-Demerdash Ebtehal. Non-alcoholic fatty liver disease: An overview of risk factors, pathophysiological mechanisms, diagnostic procedures, and therapeutic interventions[J]. Life Sciences, 2021, 271:

[8] Chi Xiaowei. Pathogenesis and drug development progress of non-alcoholic fatty liver disease[J]. Chinese Pharmacists, 2021, 24 (07): 338-343.

[9] You Suning, Gu Zhiyan, Yang Binghui, etc. Nonalcoholic fatty liver disease prevention and treatment guidelines (2018 update) [J]. Chinese Journal of Liver Diseases, 2018, 26 (3): 195-203.

[10] Fan Jiangao. Nonalcoholic fatty liver disease diagnosis and treatment guidelines[J]. Chinese Journal of Internal Medicine, 2010, 19 (6): 1-20.

[11] Li Junxiang, Chen Ju, Wang Yunliang, etc. Consensus on diagnosis and treatment of nonalcoholic fatty liver disease with integrated traditional Chinese and western medicine (2017)[J]. Chinese Journal of Integrated Traditional Chinese and Western Medicine Digestion, 2017, 29 (11): 805-811.

[12] Role of bariatric surgery in treatment of non-alcoholic fatty liver disease[J]. Journal of Contemporary Medical Sciences, 2017, 3(12).

[13] Amir Bashiri, Dinushan Nesan, Ghazaleh Tavallaee, Ian Sue-Chue-Lam, Kevin Chien, Graham F. Maguire, Mark Naples, Jing Zhang, Lilia Magomedova, Khosrow Adeli, Carolyn L. Cummins, Dominic S. Ng. Cellular cholesterol accumulation modulates high fat high sucrose (HFHS) diet-induced ER stress and hepatic inflammasome activation in the development of non-alcoholic steatohepatitis[J]. BBA-Molecular and Cell Biology of Lipids, 2016, 1861(7).

[14] Majumdar Avik, Verbeek Jef, Tsochatzis Emmanuel A. Non-alcoholic fatty liver disease: Current therapeutic options[J]. Current opinion in pharmacology, 2021, 61:

[15] Zhang Yang, Wu Weidong, Zhao Yue, Ding Yueyue, Cheng Ting, Ren Jing, Xu Zixin, Wang He. Research 
progress of treating nonalcoholic fatty liver from spleen[J]. Liaoning Journal of Traditional Chinese Medicine, 2021, 48(11): 203-206.

[16] Xie Jianhuan, Zhang Tonghai, Liu Chunfeng, Li Dongchun. Jianpi Xiaozhi Decoction in the treatment of spleen deficiency and dampness type of nonalcoholic fatty liver disease curative effect observation[J]. Yunnan Journal of Traditional Chinese Medicine, 2021, 42(05): 42-44.

[17] Huang Hongna, Fu Yanqing, Huang Jingjing, Zhang Wenfu. Progress in TCM treatment of non-alcoholic fatty liver disease[J]. Liaoning Journal of Traditional Chinese Medicine, 2020, 47(11): 201-203.

[18] Liu Lianxuan, Wu Wei, Pang Linlin, Song Zhiqi, Shi Chenxu, Yang Guanlin, Zhang Huiyong. Research progress on chemical constituents, pharmacological effects and clinical application of Gegenqinlian Decoction[J/OL]. Chinese Journal of Traditional Chinese Medicine: 1-15[2022-01-23].

[19] Chen Q, Liu M Y, Yu H Y, et al. Scutellariabaicalensis regulates FFA metabolism to ameliorate NAFLD through the AMPK-mediated SREBP signaling pathway[J]. J Nat Med, 2018, 72(3): 655-666.

[20] Yang Yuhe, Xu Xuejiao, Li Chenxue, Bai Yu, Che Zhiyuan. New progress in chemical constituents and pharmacological effects of Radix Paeoniae Rubra[J]. Chemical Engineer, 2021, 35 (09): 42-44+31.

[21] Zhou Honglin, Ma Cheng, Wang Cheng, Gong Lihong, Zhang Yafang, Li Yunxia. Research progress in use of traditional Chinese medicine monomer for treatment of non-alcoholic fatty liver disease[J]. European Journal of Pharmacology, 2021, 898 (prepublish). 